\title{
Estilo de vida y nivel de actividad física en estudiantes de residencia médica
}

Lifestyle and physical activity level in medical residence students

Sandra Jimena-Jácome ${ }^{1 \text { CvLAC }}$, Andrés Villaquirán-Hurtado CvLAC, María del Mar Meza-Cabrera ${ }^{3 凶}$

Fecha correspondencia:

Recibido: octubre 23 de 2018.

Revisado: febrero18 de 2019.

Aceptado: marzo 15 de 2019.

Forma de citar:

Jimena-Jácome S, VillaquiránHurtado A, Meza-Cabrera M del M. Estilo de vida y nivel de actividad física en estudiantes de residencia médica. Rev CES Med 2019; 33(2): 78-87.

Open access
$\frac{\text { C Derecho de autor }}{\text { Licencia creative commons }}$
$\frac{\text { Ética de publicaciones }}{\text { Revisión por pares }}$
$\frac{\text { Gestión por Open Journal System }}{\text { D0l: http://dx.doi.org/10.21615/ }}$
cesmedicina.33.2.1
ISSN 0120-8705
e-ISSN 2215-9177

Sobre los autores:

1. Magíster en Ciencias de la Educación. Grupo investigación Movimiento Corporal Humano y Calidad de Vida, Universidad del Cauca.

Comparte

70G.

\section{Resumen}

Introducción: la actividad física y un estilo de vida saludable pueden ser factores protectores de enfermedades no trasmisibles. Tanto profesionales médicos como estudiantes de Medicina juegan un rol fundamental en la adopción de una vida activa y sana por parte de sus pacientes. El objetivo del estudio fue identificar el nivel de actividad física y estilo de vida en estudiantes de residencia médica de una universidad pública. Métodos: estudio descriptivo realizado en 39 estudiantes de residencia médica quienes diligenciaron el cuestionario internacional de actividad física IPAQ y el test Fantástico para medir su estilo de vida. Resultados: el 53,8 \% de los residentes presentaban sobrepeso, 51,3 \% tenían un porcentaje de grasa muy alto, $48 \%$ de los participantes presentaba un nivel de actividad física bajo y $46 \%$ presentaba un estilo de vida adecuado. Conclusiones: los aspectos encontrados entre los residentes sugieren la toma de medidas y de decisiones oportunas para disminuir el riesgo de padecer enfermedades no transmisibles.

Palabras clave: Residencia; Estudiante; Enfermedades crónicas; Estilo de vida; Ejercicio.

\begin{abstract}
Introduction: Physical activity and a healthy lifestyle can be a protective factor for non communicable diseases; both medical professionals and medical students play a fundamental role adopting an active and healthy lifestyle and being capable to teach this behavior to their patients. The objective of the study was to identify the physical activity level and lifestyle in medical residence students from a public university. Methods: a descriptive study was carried out in 39 medical students, who completed the International Physical Activity Questionnaire (IPAQ) questionnaire and a FANTASTIC test to measure lifestyle. Results: $53.8 \%$ of the residents were overweight, $51.3 \%$ had a very high fat percentage, $48 \%$ of the participants had a low physical activity level and $46 \%$ had an adequate lifestyle. Conclusions: Medical residency students have acceptable lifestyle levels with low levels of physical activity, presence of overweight and high percentages of fat, aspects that suggest to take measures and timely decisions to reduce the risk of non-communicable diseases development.
\end{abstract}

Keywords: Residence; student; Chronic diseases; Lifestyle; Exercise. 
2. Magíster en Intervención Integral en el deportista. Grupo investigación Movimiento Corporal Humano y Calidad de Vida, Universidad del Cauca.

3. Especialista en Cirugía General. Docente departamento de Ciencias quirúrgicas Universidad del Cauca.

A pesar de conocer la importancia de la educación en el paciente, algunos estudios demuestran que no todos los pacientes reciben por parte del médico la asesoría adecuada en temas relacionados con actividad física y alimentación.

\section{Introducción}

Las enfermedades no trasmisibles como el cáncer, la diabetes, las enfermedades cardiovasculares y pulmonares afectan considerablemente la salud de la población mundial, siendo la primera causa de muerte en el mundo. Entre los factores asociados para su aparición se encuentran la obesidad, los estilos de vida no saludables y la inactividad física (1-3).

En Colombia, entre 2005 y 2013, la principal causa de muerte en la población general fue producida por enfermedades del sistema circulatorio y dentro de estas las enfermedades isquémicas del corazón responden por el 48,9\%; adicionalmente, se presentan más de dos millones de casos de hipertensión arterial en personas afiliadas al Sistema General de Seguridad Social en Salud y se reporta una prevalencia de $8,5 \%$ de diabetes mellitus $(\underline{4}, \underline{5})$.

Las conductas poco saludables están fuertemente asociadas con la aparición de este tipo de enfermedades y tales comportamientos pueden aumentarse en la vida universitaria por la falta de tiempo y las exigencias académicas, tal como ocurre durante las residencias médicas debido a las extensas horas laborales académico-asistenciales, las cuales se acompañan de situaciones estresantes producidas por la alta carga de pacientes, las diferentes enfermedades que deben atender y la preparación que deben demostrar para dar una respuesta efectiva $(\underline{2}, \underline{6}, \mathbf{7})$.

La residencia médica es un término registrado desde 1952 con el fin de cualificar y especializar médicos con competencias y habilidades específicas con un alto grado de complejidad (ㅇ). Este tipo de estudios avanzados se desarrolla con una alta carga hospitalaria, jornadas laborales extensas, disminución de las horas del sueño y alto desgaste físico y mental, que conllevan a cambios en la vida personal, en el estilo y calidad de vida de los estudiantes (9-11).

Contradictoriamente, el médico es uno de los principales actores en la consecución de prácticas más saludables por parte de sus pacientes: realizar recomendaciones en cuanto a la actividad física, alimentación saludable y manejo del peso corporal, son algunas de las indicaciones más relevantes para el tratamiento y la prevención de las enfermedades no trasmisibles (12).

A pesar de conocer la importancia de la educación en el paciente, algunos estudios demuestran que no todos los pacientes reciben por parte del médico la asesoría adecuada en temas relacionados con actividad física y alimentación $(\underline{13}, 14)$. Así mismo, los pacientes encuentran consejos más creíbles y motivadores en médicos físicamente activos y con estilos de vida saludables (14).

Teniendo en cuenta lo anterior y encontrándose pocas investigaciones en estudiantes de especialidades médicas, el objetivo del estudio fue identificar el nivel de actividad física y estilo de vida en estudiantes de residencia médica de una universidad pública de la ciudad de Popayán- Colombia.

\section{Métodos}

Se realizó un estudio descriptivo de corte transversal, con residentes de diferentes especialidades médicas: ginecología, cirugía general, anestesiología, pediatría, medicina familiar y medicina interna. Se tomó una población de 39 residentes que 
Cada uno de los participantes diligenció el Cuestionario Internacional sobre Actividad Física (International Physical Activity Questionnaire, IPAQ) y el estilo de vida por medio del test Fantástico. cumplieron los criterios de inclusión: encontrarse activos en el sistema de registro académico del periodo académico del año 2017 y manifestar aceptar la participación en el estudio.

A cada participante se le explicó el objetivo y la metodología de la investigación, se resolvieron las dudas y luego cada uno procedió a firmar el consentimiento informado. Posteriormente, se realizó la recolección de datos sociodemográficos obtenidos por auto-reporte y la obtención de las variables antropométricas. Para esta última, el sujeto debía estar con el mínimo de ropa posible para tomar la talla y el peso por medio de un tallímetro y báscula mecánica Detecto ${ }^{\circledR}$. Posteriormente, se realizó la toma de pliegues cutáneos por medio de un plicómetro Slim Guide ${ }^{\circledR}$ previamente calibrado con un material rígido de medida estándar y se finalizó con la toma del perímetro abdominal con una cinta métrica de 1,50 metros D\&T® ${ }^{\circledR}$ DGT ${ }^{\circledR}$ Tape measure. Las medidas fueron tomadas por una persona capacitada en medidas antropométricas y teniendo como referente las recomendaciones de la International Society for the Advancement of Kinanthropometry (ISAK).

Para la obtención del porcentaje de densidad corporal se aplicó la formula Durnin/ Womersley: $D C=C$-[M* Log (Suma de los pliegues)] y posteriormente se determinó el porcentaje de grasa (\%G) mediante la densidad corporal aplicando la fórmula: $\% G=[(4,95 / D C)-4,5] * 100 ;$ donde, la suma de pliegues: bíceps + tríceps + subescapular + suprailíaco del lado derecho y C y M son constantes que dependen de la edad (mayor o igual a 19 años) y el sexo $(\underline{15}, \underline{16})$.

Después de la evaluación antropométrica, cada uno de los participantes diligenció el Cuestionario Internacional sobre Actividad Física (International Physical Activity Questionnaire, IPAQ) desarrollado por la O.M.S., que permite medir el nivel de actividad física por medio de cinco objetivos de actividad independientemente. Este cuestionario ha sido recomendado y validado en 12 países diferentes para propósitos de monitoreo o investigación en población adulta entre los 15 y 69 años (17). Su calificación permite generar tres categorías que van de 1 a 3, siendo 1 la más alta, 2 moderada y 3 baja. Se decidió utilizar este cuestionario para ampliar el conocimiento de los niveles de actividad física en diferentes dominios relacionados con el trabajo, el transporte, actividades domésticas y de ocio.

Por último, se evaluó el estilo de vida de cada uno de los participantes por medio del test Fantástico (18) que permite identificar y medir el estilo de vida de una población particular. Este cuestionario contiene 25 ítems cerrados, con los que se exploran 10 dimensiones de acuerdo al acrónimo de: F: familiares y amigos, A: asociatividad y actividad física, N: nutrición, T: toxicidad A: alcohol, S: sueño y estrés, T: tipo de personalidad y actividades, I: imagen interior, C: control de la salud y sexualidad y, 0 : orden (19). Su calificación permite generar cinco categorías que permiten resumir el estilo de vida evaluado: estar en zona de peligro, algo bajo, adecuado, buen trabajo y felicitaciones.

Para el procesamiento de la información se emplearon los programas PSPP y R wizard, ambos de uso libre. Se realizó análisis descriptivo con distribuciones de frecuencia y porcentaje y, posteriormente, un análisis inferencial para establecer la relación entre las variables antropométricas, sociodemográficas y estilo de vida, para lo cual se utilizó la prueba paramétrica de T test previa verificación de requisitos de distribución normal y homogeneidad de varianzas (Shapiro Wilk y Levene, respectivamente, con $\mathrm{p} \geq 0,05)$ entre las variables índice cintura cadera y sumatoria 
El 51,3\% tenían un porcentaje de grasa muy alto y riesgo cardiovascular de acuerdo con el índice cintura cadera. de pliegues; también se utilizó la prueba no paramétrica de $U$ de Mann Whitney y la prueba exacta de Fisher para variables cualitativas y cuantitativas que no cumplieron requisitos.

Adicionalmente, se realizaron pruebas de correlación y se calculó de manera indirecta el riesgo mediante el odds ratio (O.R.). Todas las pruebas se tomaron con un nivel de confianza del $95 \%$, a dos colas, considerando valor estadísticamente significativo la $p$ menor de 0,05.

Para la realización de la investigación se tuvieron en cuenta los aspectos éticos de la Declaración de Helsinki (20) y la resolución 8430 del Ministerio de Salud (21), clasificando esta investigación en un nivel de riesgo mínimo, además este estudio contó con el aval del comité de ética de la Universidad del Cauca.

\section{Resultados}

La edad promedio fue de 29 años de edad, hubo mayor frecuencia de hombres (64,1\%), el 17,9 \% estudiaba y trabajaba al mismo tiempo. El 53,8 \% presentaban sobrepeso, siendo más frecuente en los hombres. El 51,3 \% tenían un porcentaje de grasa muy alto y riesgo cardiovascular de acuerdo con el índice cintura cadera (cuadro 1)

Cuadro 1. Características sociodemográficas y antropométricas de los participantes

\begin{tabular}{|c|c|}
\hline Variable & $\%$ \\
\hline \multicolumn{2}{|l|}{ Años } \\
\hline $20-29$ & 61,5 \\
\hline $30-39$ & 38,5 \\
\hline \multicolumn{2}{|l|}{ Sexo } \\
\hline Masculino & 64,1 \\
\hline Femenino & 35,9 \\
\hline \multicolumn{2}{|l|}{ Estrato } \\
\hline 1 & 2,6 \\
\hline 2 & 12,8 \\
\hline 3 & 30,8 \\
\hline 4 & 35,9 \\
\hline 5 & 15,4 \\
\hline 6 & 2,6 \\
\hline \multicolumn{2}{|l|}{ Ocupación } \\
\hline Además de estudiar trabaja & 17,9 \\
\hline Sólo estudia & 82,1 \\
\hline \multicolumn{2}{|l|}{$I M C$} \\
\hline Peso normal & 46,2 \\
\hline Sobrepeso & 53,8 \\
\hline \multicolumn{2}{|l|}{ Porcentaje graso } \\
\hline Recomendado & 7,7 \\
\hline Alto & 41 \\
\hline Muy alto & 51,3 \\
\hline \multicolumn{2}{|l|}{ Riesgo cardiovascular } \\
\hline Si & 51,3 \\
\hline No & 48,7 \\
\hline \multicolumn{2}{|l|}{ Con riesgo cardiovascular } \\
\hline Hombres & 20,5 \\
\hline Mujeres & 30,8 \\
\hline
\end{tabular}


El 48,7\% presentó bajo nivel de actividad física. Quienes presentaban riesgo cardiovascular mostraron un mayor peso corporal $(p=0,000)$, así como mayor tejido adiposo calculado por la sumatoria de pliegues $(p=0,000)$.
El 48,7 \% presentó bajo nivel de actividad física y 43,6 \% tenían un estilo de vida adecuado (cuadros $\underline{2}$ y $\underline{3}$ ). Quienes presentaban riesgo cardiovascular mostraron un mayor peso corporal $(p=0,000)$, así como mayor tejido adiposo calculado por la sumatoria de pliegues $(p=0,000)$ (grafico 1).

Cuadro 2. Clasificación de IPAQ según sexo, estrato socioeconómico y estado civil

\begin{tabular}{cccc}
\hline & \multicolumn{3}{c}{ Clasificación IPAQ } \\
\hline & Alta & Moderada & Baja \\
& $\%$ & $\%$ & $\%$ \\
\hline Sexo & & & \\
\hline Masculino & 7,6 & 23,1 & 30,8 \\
Femenino & 15,4 & 5,1 & 18 \\
\hline Estrato & & & \\
\hline 1 & 0 & 2,6 & 0 \\
2 & 7,7 & 2,6 & 2,6 \\
3 & 5,1 & 18 & 23 \\
4 & 10,2 & 2,6 & 12,7 \\
5 & 0 & 2,6 & 2,6 \\
\hline 6 & 0 & 0 & \\
\hline Estado civil & & & 18 \\
\hline Soltero & 15,3 & 20,5 & $\mathbf{4 8 , 7}$ \\
\hline Casado & 5,1 & 7,7 & \\
\hline Unión libre & 2,6 & 0 & $\mathbf{2 8 , 2}$ \\
\hline Total & $\mathbf{2 3}$ & & \\
\hline
\end{tabular}

Cuadro 3. Estilo de vida usando el cuestionario Fantástico

\begin{tabular}{lc}
\hline \multicolumn{1}{c}{ Variable } & $\%$ \\
\hline Malo (47-72) & 20,5 \\
\hline Regular (73-84) & 43,6 \\
\hline Bueno (85-102) & 35,9 \\
\hline Excelente (103-120) & 0 \\
\hline
\end{tabular}




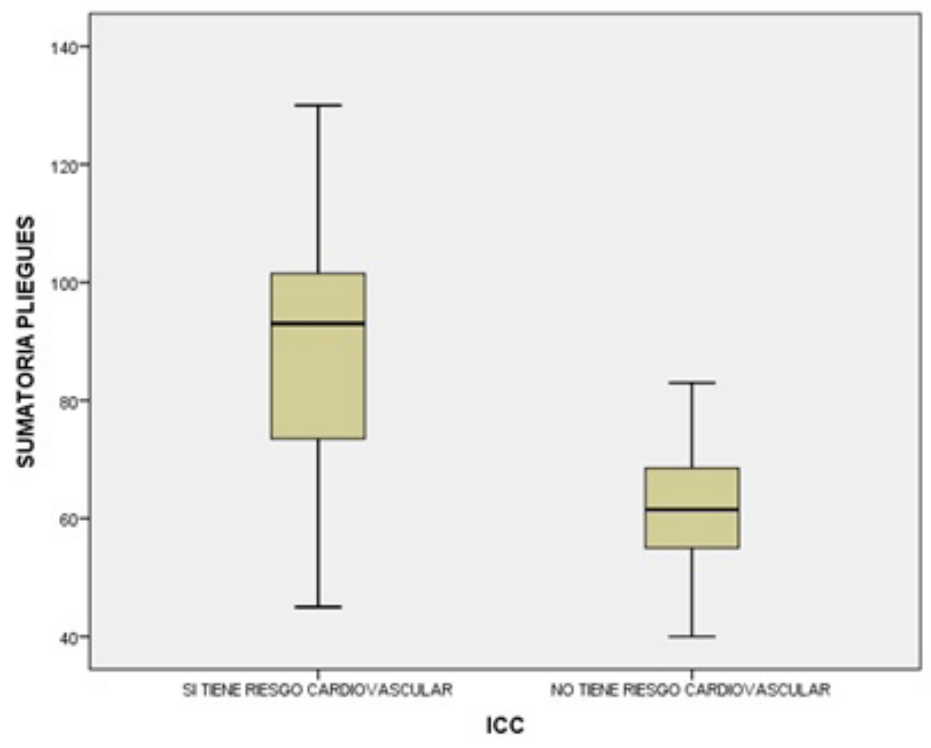

Grafico 1. Distribución del Índice cintura cadera según sumatoria de pliegues

Se encontró asociación entre el índice cintura cadera y el sexo, índice cintura cadera e índice de masa corporal y entre índice cintura cadera y estilo de vida.
Cuadro 4. Relación entre índice cintura cadera, sexo, índice de masa corporal y estilo de vida

\begin{tabular}{lccccc}
\hline \multicolumn{5}{c}{ Con riesgo } & \multicolumn{2}{c}{ Sin riesgo } & $\boldsymbol{P}$ \\
\hline \multicolumn{1}{c}{$\boldsymbol{n}$} & $\boldsymbol{\%}$ & $\boldsymbol{n}$ & $\mathbf{\%}$ & \\
\hline Sexo & 16 & 66,7 & 8 & 33,3 & 0,005 \\
Masculino & 3 & 20,0 & 12 & 80,0 & \\
Femenino & & & & & \\
\hline IMC & 15 & 71,4 & 6 & 28,6 & 0,002 \\
Sobrepeso & 4 & 22,2 & 14 & 77,8 & \\
Normal & & & & & \\
\hline Estilo de vida & 6 & 85,7 & 1 & 14,3 & \multirow{2}{*}{0,047} \\
Algo bajo & 9 & 50,0 & 9 & 50,0 & \\
Adecuado & 4 & 28,6 & 10 & 71,4 & \\
Buen camino & $\mathbf{1 9}$ & $\mathbf{4 8 , 7}$ & $\mathbf{2 0}$ & $\mathbf{5 1 , 3}$ & \\
\hline Total & & & & & \\
\hline
\end{tabular}

\section{Discusión}

El bienestar de una persona incluye medidas que buscan promover aspectos relacionados con una óptima salud física, mental, social y psico-afectiva, por medio de estrategias facilitadoras de entornos saludables, fomentando la adquisición de estilos de vida saludables y la prevención de enfermedades no trasmisibles, para el caso del estudiante se busca favorecer los procesos de aprendizaje que mejoren posteriormente el actuar personal, profesional y la calidad de vida del futuro médico especialista (12). 
Mayo - agosto de 2019 - Pág 84

Los bajos niveles de actividad física pueden estar asociados a la falta de oportunidades que tienen los estudiantes dentro de los programas de formación, dada la disponibilidad restringida de tiempo y la sobrecarga académica.
Un aspecto para evaluar y que identifica factores de riesgo de enfermedades no transmisibles es el índice de masa corporal. Al igual que en otras investigaciones, el sobrepeso aparece con mayor frecuencia en los estudiantes de residencia $(12,22)$; sin embargo, estos hallazgos no concuerdan en relación con el sexo, puesto que, a diferencia del presente estudio, otros autores reportan que las mujeres presentan más prevalencia de sobrepeso (23). Un índice de masa corporal elevado es considerado un factor de riesgo para presencia de enfermedades crónicas (24).

Más de la mitad de estudiantes presentó un índice cintura cadera por encima de lo normal y mayor tejido adiposo, lo que puede estar relacionado con los comportamientos negativos en su alimentación y la falta de autorregulación por parte de los estudiantes para modificar su exceso de peso (25). Por el contrario, el control de los niveles de grasa, sumado a una dieta rica en vegetales, frutas y verduras, bajo consumo de alcohol y estar físicamente activo se relacionan con un riesgo muy bajo de sufrir enfermedades cardiovasculares y diabetes mellitus (2 $\underline{6})$.

Referente al nivel de actividad física, los resultados encontrados en estos residentes son preocupantes, presentando un bajo nivel en dicho hábito, siendo paradójico con el conocimiento que deben tener respecto a los beneficios de la práctica cotidiana de ejercicio físico. Al respecto Mora et al., reportan que el 41,9\% de estudiantes de postgrado en Medicina son sedentarios (12), muy similar a los encontrados en esta investigación. Otros estudios difieren, encontrando niveles más altos de actividad física, como el reportado en estudiantes universitarios españoles, en donde el 68,4 \% de hombres y el $48,4 \%$ de mujeres practican actividades deportivas frecuentemente (27); además, los mayores niveles de actividad física encontrados pertenecen a los estudiantes de sexo masculino (28).

Estos bajos niveles de actividad física pueden estar asociados a la falta de oportunidades que tienen los estudiantes dentro de los programas de formación (29), dada la disponibilidad restringida de tiempo y la sobrecarga académica; sin embargo, el conocimiento de estas razones puede ser motivo de estudio en próximas investigaciones.

Los bajos niveles de actividad y su relación con los bajos estratos socioeconómicos son reportados por otros estudios (30), que encuentran desigualdades en los dominios de actividad física para el aprovechamiento del tiempo libre, uso de la bicicleta y caminar como medios de transporte y evidenciando diferencias por sexo y nivel socioeconómico para cumplir las tareas necesarias para ser físicamente activos. El presente estudio muestra que los residentes de sexo femenino son quienes presentan mayores niveles de actividad física.

En cuanto al estilo de vida, un gran porcentaje presentó resultados adecuados, aunque otros estudios reportan un mal estilo de vida, debido a una práctica de actividad física muy irregular y hábitos de alimentación deficientes (23). Un estilo de vida inadecuado se puede presentar debido a comportamientos sedentarios, como jugar videos juegos, navegar en redes sociales y ver televisión en el tiempo libre $(\underline{2}, \underline{31})$, aunque se conoce que conductas sanas como realizar ejercicio, mantener una buena salud mental y comer saludablemente, permiten tener una adecuada calidad de vida $(\underline{7}, \underline{31})$.

\section{Conclusiones}

Los estudios de especialización medica implican una actividad laboral exigente, generada por una gran demanda de tiempo y dedicación, limitando el tiempo libre y aumentando la posibilidad de tener estudiantes sedentarios con comportamientos 
Es importante incluir en los programas académicos, la formación específica del estudiante en aspectos relacionados con estilo de vida activos, así como también brindar los espacios necesarios y suficientes para su práctica. poco saludables, los cuales pueden afectar la salud del estudiante y la aparición de enfermedades no trasmisibles. Por esta razón, es importante incluir en los programas académicos, la formación específica del estudiante en aspectos relacionados con estilo de vida activos, así como también brindar los espacios necesarios y suficientes para su práctica.

En la población de estudio, existe una clara asociación entre el nivel de estilo de vida y el riesgo cardiovascular que podría ser de utilidad en futuros estudios de intervención para establecer metodologías que permitan mejorar el estilo de vida y disminuir el riesgo cardiovascular.

\section{Declaración de autores}

Se declara no tener conflicto de intereses.

\section{Agradecimientos}

Agradecemos a la Universidad del Cauca, a los estudiantes y docentes del programa de Fisioterapia por su colaboración y compromiso con la investigación.

\section{Bibliografía}

1. Tovar G, López G, Ibáñez M, Alvarado R, Lobelo F, Duperly J. Institutionalized physical activity curriculum benefits of medical students in Colombia. Educ Heal Chang Learn Pract. 2016;29(3):203-9.

2. Yan H, Zhang R, Oniffrey T, Chen G, Wang Y, Wu Y, et al. Associations among Screen Time and Unhealthy Behaviors, Academic Performance, and Well-Being in Chinese Adolescents. Int J Environ Res Public Health. 2017;14(6):1-15. 3. $\quad$ K i m $\mathrm{H}$, Oh S. Noncommunicable diseases: Current status of major modifiable risk factors in Korea. J Prev Med Public Heal. 2013;46(4):165-72.

3. Vargas-Uricoechea H, Casas-Figueroa L. Epidemiología de la diabetes mellitus en Sudamérica: la experiencia de Colombia. Clínica e Investig en Arterioscler. 2016;28(5):245-56.

4. Ministerio de Salud y Protección Social. Direccion de Epídemiologia y Demografia. Analisis de la Situacion de Salud (ASIS) COLOMBIA. Inst Nac Salud. 2015.

5. Macías F, Malmusi D, Olabarría M, Borrell C. Cardiometabolic risk inequalities in Colombia. Int J Cardiol. 2017;202(2016):156-8.

6. Mehmood Y, Al-Swailmi F, Al- Enazi S. Frequency of obesity and comorbidities in medical students. Pak Med Sci. 2016;32(6):1528-32.

7. Duque G, Ortiz L. Sistema de residencias médicas en Colombia: Marco conceptual para una propuesta de regulación. 2013; 1-64.

8. Prieto-Miranda S, Rodriguez G, Jiménez C, Guerrero- Quintero L. Desgaste profesional y calidad de vida en médicos residentes. Rev Med Inst Seguro Soc. 2013;51(5):574- 9

9. Álvarez-Hernández G, Medécigo-Vite S, Ibarra-García C. Prevalencia del síndrome de desgaste profesional en médicos residentes de un hospital pediátrico en el Estado de Sonora. Rev Bol Med Infant Mex.2009; 67(1):44-51. 
10. Miní E, Medina J, Rojas L, Peralta V, Butron J, Gutierrez E. Programa de residentado médico: percepciones de los médicos residentes en hospitales de Lima y Callao. Rev Peru Med Exp Salud Publica. 2015;32(2):303-10.

11. Mora SL, Moreno CA. Autopercepción de salud y de red de apoyo y funcionalidad familiar, de los estudiantes que inician posgrados de medicina. Repert Med y Cirugía. 2016;25(1):8-14.

12. Sinclair J, Lawson B, Burge F. Which patients receive advice on diet and exercise? Do certain characteristics affect whether they receive such advice?. Can Fam Physician. 2008;54(3):404-12.

13. Frank E, Tong E, Lobelo F, Carrera J, Duperly J. Physical activity levels and counseling practices of U.S. medical students. Med Sci Sports Exerc. 2008;40(3):413-21.

14. Stewart A, Marfell-Jones M, Olds T. Protocolo Internacional para la Valoración Antropométrica ISAK. 2011.

15. Douglas T, Kennedy M, Quirk M, Yi S, Singh R. Accuracy of Six Anthropometric Skinfold Formulas Versus Air Displacement Plethysmography for Estimating Percent Body Fat in Female Adolescents with Phenylketonuria. JIMD Reports. 2012;4:113-16.

16. Wanner M, Probst-Hensch N, Kriemler S, Meier F, Autenrieth C, Martin BW. Validation of the long international physical activity questionnaire: Influence of age and language region. Prev Med Reports. 2016;3:250-6.

17. Betancurth D, Velez C, Jurado L. Validación de contenido y adaptación del cuestionario Fantastico por técnica Delphi. Rev Salud Uninorte. 2015;31(2):214-27.

18. Ramírez-Vélez R, Agredo R. Fiabilidad y validez del instrumento "Fantástico" para medir el estilo de vida en adultos colombianos. Rev Salud Publica. 2012;14(2):226-37.

19. Ministerio de Salud. Resolución Nº08430 de 1993, octubre 4, por la cual se establecen las normas científicas, técnicas y administrativas para la investigación en salud. 1993. Disponible en: https://www.minsalud.gov. co/Normatividad_ Nuevo/RESOLUCION \%208430 \%20DE \%201993.pdf..

20. Di Ruggiero M. Declaración de Helsinki, pricipios y valores bioéticos en juego en la investigación Medica con seres humanos. Revista Colombiana de Bioética. 2011;6(1):125-44.

21. Alodhayani A, Alshaikh O, Ghomraoui F, AlShaibani T, Algadheeb A, Bendahmash $A$, et al. Correlation between obesity and sleep disturbance in Saudi medical students. J Phys Ther Sci [Internet]. 2017;29(2):181-6.

22. Al-Qahtani MH. Dietary Habits of Saudi Medical Students at University of Dammam. Int J Health Sci. 2016;10(3):353-62. 
23. Saeed E, Assiri AM, Awadeljack I, Aljasser AS, Alhuzimi AM, Assiri AA, et al. Obesity and associated risk factors among students of health colleges of king saud university, Saudi Arabia: A cross-sectional study. J Pak Med Assoc. 2017;67(3):355-9.

24. Arguello-González A, Cruz-Arteaga G. Autoregulación de habitos alimenticios en médicos residentes de Medicina Familiar con sobrepeso u obesidad. Aten Fam. 2017; 24(4): 160-64.

25. Si J, Yu C, Guo Y, Bian Z, Li X, Yang L, et al. Adherence to a healthy lifestyle and the risk of type 2 diabetes in Chinese adults. International Journal Epidemiology. 2017;60(5):836-42.

26. Romaguera D, Tauler P, Bennasar M, Pericas J, Moreno C, Martinez S, et al. Determinants and patterns of physical activity practice among Spanish university students. J Sports Sci. 2011;29(9):989-97.

27. Bergier J, Bergier B, Tsos A. Variations in physical activity of male and female students from the Ukraine in health-promoting life style. Ann Agric Environ Med. 2017;24(2):217-21.

28. Strong A, Stoutenberg M, Hobson-Powell A, Hargreaves M, Beeler H, Stamatakis E. An evaluation of physical activity training in Australian medical school curricula. J Sci Med Sport. 2017;20(6):534-8.

29. González S, Sarmiento O, Lozano O, Ramírez A, Grijalba C. Niveles de actividad física de la población colombiana: desigualdades por sexo y condición socioeconómica. Biomédica. 2014; 34(3)447-59.

30. Khan MA, Shabbir F, Rajput TA. Effect of gender and physical activity on internet addiction in medical students. Pakistan J Med Sci. 2017;33(1):191-4.

31. Angkurawaranon C, Jiraporncharoen W, Sachdev A, Wisetborisut A, Jangiam W, Uaphanthasath R. Predictors of quality of life of medical students and a comparison with quality of life of adult health care workers in Thailand. Springer Plus. 2016;5:584. 